\section{CATASTROPHIC ANTIPHOSPHOLIPID SYNDROME: WHAT IS NEW?}

Ricard Cervera. Dept. of Autoimmune Diseases, Hospital Clínic, Barcelona, Catalonia, Spain

10.1136/lupus-2020-eurolupus. 11

Background Catastrophic antiphospholipid syndrome (CAPS) is a rare variant that accounts for $1 \%$ of patients with antiphospholipid syndrome.

Methods The current knowledge of this potential devastating entity comes from the International Registry of patients with CAPS, named 'CAPS Registry'. This presentation shows the results of the most recent analysis of the registry.

Results Small vessel thrombosis, laboratory features of microangiopathic haemolytic anemia, and development of multisystem involvement in a very short period of time are the main characteristics of this syndrome. Clinical manifestations are due to thrombosis but also, although the evidences are indirect, to excess of proinflammatory cytokines. Therefore, treatment strategy is based on the combination of anticoagulation, glucocorticoids, plasma exchange and/or intravenous immunoglobulins, the so-called triple therapy. In refractory cases or in those with initial life-threatening situation, rituximab may be an effective option. Recently, some cases of CAPS have been effectively treated with the addition of eculizumab to the triple therapy.

Conclusions Despite its low frequency, the mortality-related is still very high, ranging from $50 \%$ of patients in the first series to $37 \%$ in the most recent data.

\section{RITUXIMAB FOR REFRACTORY MANIFESTATIONS OF THE ANTIPHOSPHOLIPID SYNDROME - THE ISRAELI EXPERIENCE}

\begin{abstract}
1,2Nancy Agmon-Levin, 2,3Mark Berman, 2,4 Liora Harel, 1,2Soad Haj Yahia, ${ }^{2,5}$ Merav Lidar, 2,3 Daphna Paran. 'Clinical Immunology, Angioedema and Allergy Unit, Center for Autoimmune Diseases, Sheba Medical Center, Tel Hashomer; ${ }^{2}$ Sackler School of Medicine, Tel-Aviv University; ${ }^{3}$ Dept. of Rheumatology, Tel Aviv Medical Center; ${ }^{4}$ Pediatric Rheumatology unit, Schneider Medical Center; ${ }^{5}$ Rheumatology Unit, Center for Autoimmune diseases, Sheba Medical Center, Israel
\end{abstract}

10.1136/lupus-2020-eurolupus. 12

Background Clinical manifestations of the antiphospholipid syndrome (APS) are heterogeneous and may be difficult to treat. Evidence that B cells and particularly anti-phospholipid antibodies (aPL) are involved in APS-clinical events has been documented. Thus, the ability of rituximab (RTX) to deplete $\mathrm{B}$ cells and possibly reduce aPL titers makes it an appealing potential therapy for this autoimmune disease. Real world data on the usefulness of RTX treatment for APS is scarce. In this study we report outcomes of RTX administration in treating different manifestations of APS.

Methods In this retrospective case series, data from 3 medical centers in Israel was collected regarding the use of RTX to treat APS. Medical records were reviewed for clinical manifestations, indication for RTX treatment, concomitant medications, aPL status and the response to treatment. The latter was clinically defined as complete resolution of the indicated manifestation, partial improvement or no response.

Results We included 40 APS patients in this case series, 31 primary-APS and 9 with APS secondary to SLE. Our cohort consisted of 16 males and 24 females, mean age $39 \pm 14$ years, 29 were defined as triple-aPL positive. All patients presented with 1-2 difficult to treat manifestations including: diffuse alveolar hemorrhage $(\mathrm{DAH}-\mathrm{n}=6)$, recurrent thrombosis despite anticoagulation $(n=10)$, thrombocytopenia and/or hemolytic anemia $(n=10)$, neurological manifestations $(n=7)$, nephropathy $(n=2)$ vasculitis/leg ulcers (Skin) $(5 \mathrm{p})$ and catastrophic-APS $(\mathrm{n}=2)$. Notably, $32 \quad(80 \%)$ patients responded favorably (complete response - 22; partial improvement -10), 5 did not respond to RTX therapy and 3 patients died within 12 months (figure 1). Concomitant therapies (e.g. hydroxychloroquine, glucocorticoids, cyclophosphamide, plasmapheresis, azathioprine etc.) did not correlate with favorable responses, rather treatment with cyclophosphamide or plasmapheresis correlated with worse outcomes $(p<0.01)$. In this cohort, the rituximab protocol was linked with treatment success as 17 patients received 4 weekly doses of $375 \mathrm{mg} / \mathrm{m}^{2}$ while 23 received 2 doses of $1000 \mathrm{mg} 14$ days apart. The former protocol resulted in $17 / 17(100 \%)$ responders, $12 / 17(70 \%)$ with a complete response, while following the latter protocol 15/23 (65\%) responded, 10/23(45\%) with a complete response $(\mathrm{p}=0.012)$. A decrease in aPL titers within 2-6 months of RTX treatment correlated with a good response to rituximab therapy. Data regarding aPL titers within 2-6 months was available for 22 patients of whom 13 experienced a complete response correlating with a significant decrease in the RVVT-ratio, anti-cardiolipin-IgM and anti-beta2GPI-IgG titers $(\mathrm{p}=0.02,0.016$ and 0.015 respectively).

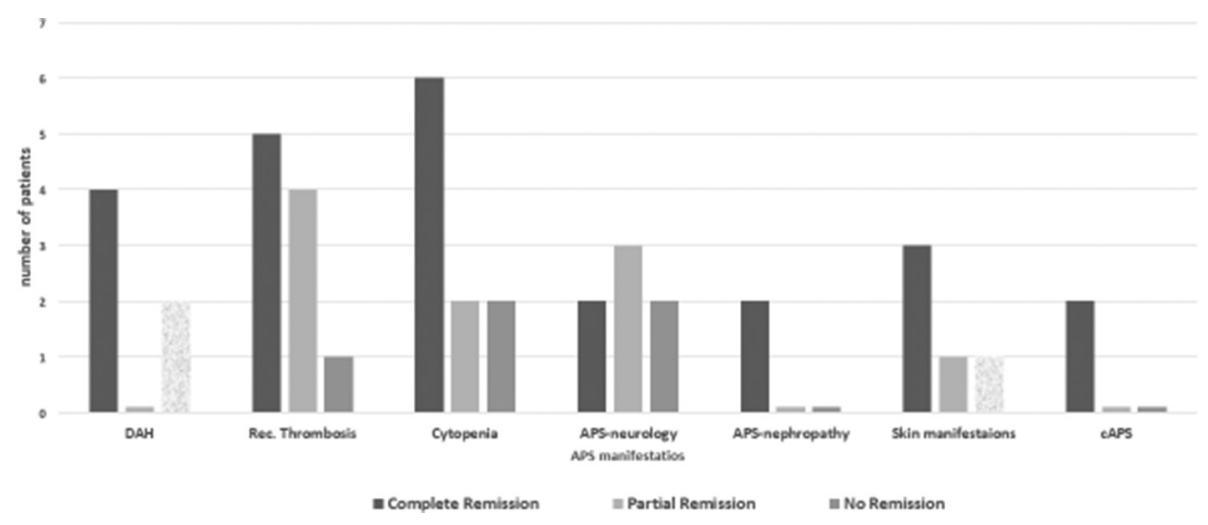

Abstract I12 Figure 1 Response of APS manifestation to rituximab treatment 